\title{
Stretching sensors by confinement
}

Making electronics more compatible with our skin is a key goal for sensor technology. Writing in Science, Zhenan Bao, Jong Won Chung and colleagues ${ }^{1}$ use nanoconfinement to generate transparent semiconductor polymer films that deform easily and retain their properties under strain. The approach is a general method for fabrication of highly stretchable semiconductor ploymers.

The challenges of making materials that meet the needs of wearable devices and their users have been approached from different angles. On the one hand, geometric designs, such as serpentine or wavy patterns and kirigami (origami but with cuts in addition to folding), have enabled stretchability by mechanical design. The drawback is that design of the resulting device and components must conform to the pattern, which imposes limitations. Alternatively, intrinsically stretchable polymers may be ideal but are hard to come by, as they tend to lack either good carrier mobility or plasticity under strain.

Bao, Chung and colleagues ${ }^{1}$ mix a semiconductor polymer with an elastomer and layer a film of this solution over a substrate. As the film dries out, the polymer segregates from the elastomer and a mesh of nanofibers self-assembles. It is this network of nanofibers that contribute to charge transport, and the morphology of the mesh allows some stretching before the polymer itself is stretched. The film can be transferred onto an elastic dielectric material for further study and to generate an electronic device.

Taking their new films and incorporating previous knowledge for construction of other
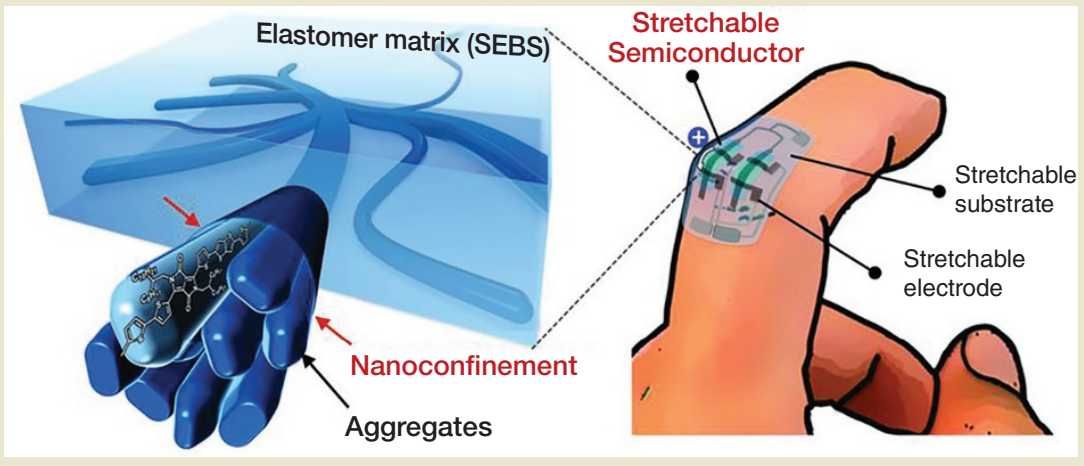

device components, the researchers build a fully stretchable wearable device with carbon nanotube networks as electrodes and a stretchable dielectric layer. The result is a soft transparent film that conforms to human skin. The device is ductile and robust - it can withstand twisting, poking and hundreds of stretching cycles, without considerable loss in its conducting capacity or evidence of cracking.

The idea to use nanoconfinement originated from work done by the polymer physics community, says Bao, a professor of chemical engineering at Stanford University. Nanoconfinement had been shown to make polymers softer, a phenomenon that had been used to compress or mold insulating polymers into various shapes and nanopatterns. "Because we are interested in stretchable materials, we started to think about approaches we could use from the fundamental polymer physics side," she says. "To achieve nanoconfinement, the molecules within the fibers have to align properly and the fibers need to connect with each other to form the right-size network. We chose the polymer and elastomer based on what we knew, but the self-assembly is hard to predict, so we were happy it worked," she recalls.

Having tried it on other semiconductor polymers successfully, the researchers have shown that the method is generalizable for the fabrication of highly stretchable polymer films. "We don't have to use very sophisticated top-down lithography or patterning technique to nanoconfine the fibers," says Bao, "so we believe it will be easily scalable." The challenge they are focusing on next is using an additive approach to layer the device components one by one on the intended substrate. "This will make the process much more relevant for making stretchable devices and also for making more complex circuits," says Bao.

Irene Jarchum, Associate Editor

1. Xu, J. et al. Highly stretchable polymer semiconductor films through the nanoconfinement effect. Science 355, 59-64 (2017).

\section{Research Highlights}

Papers from the literature selected by the Nature Biotechnology editors. (Follow us on Twitter, @NatureBiotech \#nbtHighlight)

T-cell transfer therapy targeting mutant KRAS in cancer

Tran, E. et al. N. Engl. J. Med. 375, 2255-2262 (2016)

Distribution and clinical impact of functional variants in 50,726 whole-exome sequences from the DiscovEHR study Dewey, F.E. et al. Science 354, aaf6814 (2016)

Perturb-Seq: dissecting molecular circuits with scalable single-cell RNA profiling of pooled genetic screens Dixit, A. et al. Cell 167, 1853-1866.e17 (2016)

A multiplexed single-cell CRISPR screening platform enables systematic dissection of the unfolded protein response Adamson, B. et al. Cell 167, 1867-1882.e21 (2016)

Dissecting immune circuits by linking CRISPR-pooled screens with single-cell RNA-seq Jaitin, D.A. et al. Cell 167, 1883-1896.e15 (2016) 